\title{
Uma nova espécie de Cecidomyiidae (Diptera) associada com Sebastiania glandulosa (Euphorbiaceae)
}

\author{
Valéria Cid Maia \& Uellinton Pereira de Oliveira
}

Departamento de Entomologia, Museu Nacional, Universidade Federal do Rio de Janeiro, Quinta da Boa Vista, São Cristóvão, 20940-040 Rio de Janeiro, RJ, Brasil. (maiavcid@acd.ufrj.br; uellintonoliveira@yahoo.com.br)

\begin{abstract}
A new species of Cecidomyiidae (Diptera) associated with Sebastiania glandulosa (Euphorbiaceae). Schizomyia spherica sp. nov, that induces spherical galls on Sebastiania glandulosa, is described and illustrated based on larvae, pupae, males and females from the restinga of Barra de Maricá, Rio de Janeiro, Brazil.
\end{abstract}

KEYWORDS. New species, gall, Schizomyia, taxonomy.

RESUMO. Schizomyia spherica sp. nov., que induz galhas esféricas em Sebastiania glandulosa, é descrita e ilustrada com base em larvas, pupas, machos e fêmeas de material coletado na restinga da Barra de Maricá (Rio de Janeiro, Brasil).

PALAVRAS-CHAVE. Espécie nova, galha, Schizomyia, taxonomia.

MAIA (2001) registrou a presença de galhas esféricas desenvolvidas a partir das gemas apicais e laterais em Sebastiania glandulosa (Mart.) Pax. (Euphorbiaceae) induzidas por Schizomyia sp. (Diptera, Cecidomyiidae), na restinga da Barra de Maricá e de Itaipuaçu (Maricá, RJ). Esta planta tem ampla distribuição na região Neotropical, sendo que no Brasil ocorre em Minas Gerais, Rio de Janeiro, Goiás, Mato Grosso e Mato Grosso do Sul.

Estudos morfológicos prévios indicaram tratar-se de uma espécie nova de Schizomyia Kieffer, 1889. Este gênero caracteriza-se por apresentar palpo com quatro artículos, ovipositor em forma de agulha e larva com quatro pares de papilas terminais.

Este gênero cosmopolita inclui 48 espécies descritas, seis delas da Região Neotropical: S. ipomoeae Felt, 1910 (St. Vincent), S. manihoti Tavares, 1925 (Brasil), S. macrocapillata Maia, 2005 (Brasil), S. serjaniae Möhn, 1960 (El Salvador), S. stachytarphetae Barnes, 1932 (Trinidad) e S. tиiиiu Urso-Guimarães \& Amorim, 2002 (Brasil). As espécies neotropicais ocorrem respectivamente nas seguintes plantas hospedeiras: Ipomoea sp. (Convolvulaceae) (Felt, 1910); Manihot utilissima (Euphorbiaceae) (TAVARES, 1925); Bauhinia brevipes (Fabaceae) (MAIA \& FERNANDES, 2005); Serjania goniocarpa (Sapindaceae) (MöHN, 1960); Stachytarpheta cayennensis e $S$. jamaicensis (Verbenaceae) (BARNES, 1932); e Bauhinia rufa (Fabaceae) (URSO-GUIMARÃES \& AMORIM, 2002).

Schizomyia spherica sp. nov. é a quarta espécie descrita do gênero associada com Euphorbiaceae.

\section{MATERIAL E MÉTODOS}

Parte dos exemplares examinados foram previamente incorporados na coleção de Diptera do Museu Nacional (MNRJ) por VCM, como material testemunho de um levantamento de galhas realizado em 1997, em áreas de restinga no município de Maricá, RJ,
Brasil. Material adicional foi obtido na restinga da Barra de Maricá, a partir de coleta de amostras de galhas esféricas em Sebastiania glandulosa em diversas ocasiões, por diferentes coletores, desde setembro de 2000 a março de 2006. As larvas foram obtidas através da dissecação das galhas, em laboratório, com auxílio de estilete e pinça, e foram inicialmente conservadas em álcool a $70 \%$. O restante do material foi acondicionado em potes plásticos etiquetados, tampados com tela de náilon e contendo no fundo uma camada de cerca de $10 \mathrm{~cm}$ de solo da restinga, uma vez que a larva de terceiro instar abandona a galha e enterra-se no solo, onde então transforma-se em pupa. Os potes foram vistoriados diariamente para verificação da emergência dos adultos. Exemplares de larva de terceiro instar, exúvias da pupa e imagos foram inicialmente preservados em álcool a 70\% e, posteriormente montados em lâminas permanentes para microscopia, seguindo a metodologia de GAGNÉ (1994). Todos os espécimes, incluindo os tipos, estão depositados na coleção de Diptera do Museu Nacional, Rio de Janeiro (MNRJ). O comprimento da asa foi medido a partir do árculo até o ápice da mesma. Todas as medições foram feitas utilizando-se uma lâmina com escala de 0,1 e $0,01 \mathrm{~mm}$.

\section{Schizomyia spherica sp. nov. (Figs. 1-14)}

Adulto. Comprimento do corpo: macho: 1,3-1,9 mm $(\mathrm{n}=4)$; fêmea: 2,2-2,7 mm ( $\mathrm{n}=4)$.

Cabeça (Fig. 1). Olhos com facetas circulares e muito próximas entre si. Antena: escapo cilíndrico, mais afilado na base; pedicelo globuloso, ambos com cerdas, flagelômeros cilíndricos, ornados como nas Figs. 2 e 3. Frontoclípeo com 20 cerdas; labro triangular com três pares de cerdas sensoriais ventrais; hipofaringe com a mesma forma que o labro, setulosa apicalmente (Fig. 4). Labelo ovóide, com cerdas laterais e dois pares de cerdas 
sensoriais medianas curtas (Fig. 5). Palpo com quatro artículos crescentes em comprimento, todos com cerdas.

Tórax. Anepímero com 16-35 cerdas $(n=5)$. Asas (Fig. 6). Comprimento: macho, 1,3-1,6 mm ( $\mathrm{n}=4)$; fêmea, 1,83-1,97 mm ( $\mathrm{n}=4)$. $\mathrm{R} 1$ encontrando $\mathrm{C}$ um pouco antes da metade do comprimento da asa; R5 ligeiramente curva e encontrando $\mathrm{C}$ imediatamente após o ápice da asa, M3 presente, $\mathrm{CuA}$ bifurcada, $\mathrm{CuP}$ presente. Garras tarsais simples, delgadas e levemente curvas próximo ao ápice, empódio alongado, atingindo a curvatura da garra (Fig. 7).

Abdome. Macho (Fig. 8): tergitos 1-7 regulares, com uma fileira de cerdas caudais, um par basal de sensilas tricóides e escamas no restante de sua extensão. Tergito 8 esclerotinizado, com um par basal de sensilas tricóides. Esternitos 2-6 regulares, com cerdas abundantes medianamente, cerdas caudais, um par basal de sensilas tricóides e escamas no restante de sua extensão; esternitos 7 e 8 quadrangulares, esclerotinizados, com cerdas abundantes medianamente, cerdas caudais, um par basal de sensilas tricóides e escamas no restante de sua extensão. Fêmea (Fig. 9): tergitos 1-7 como no macho; tergito 8 como na Fig. 9; esternitos 2-6 como no macho, esternito 7 ovalado,

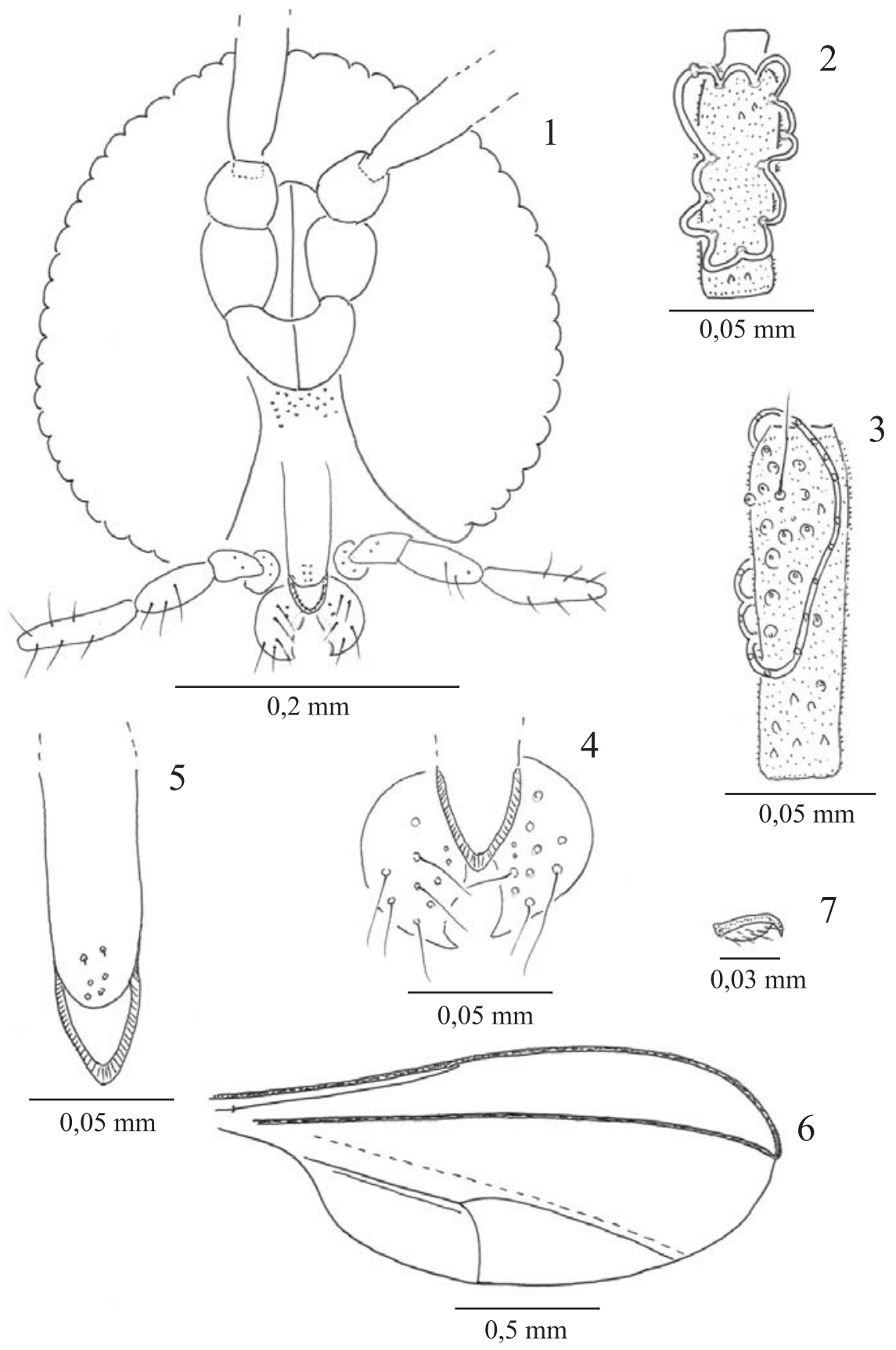

Figs. 1-7. Schizomyia spherica sp. nov.: 1, macho, cabeça, vista frontal; 2, macho, flagelômero 5; 3, fêmea, flagelômero 2; 4, fêmea, labelo, vista frontal; 5, fêmea, labro e hipofaringe, vista frontal; 6, fêmea, asa; 7, macho, perna anterior, garra e empódio. 
com cerdas distribuídas em toda a sua extensão, exceto no quinto basal; esternito 8 não-esclerotinizado.

Terminália masculina (Fig. 10). Gonocoxito estreito e alongado, cerca de três vezes mais longo que largo; gonóstilo curto, mais largo apicalmente; cerco com dois lobos reniformes cerdosos; hipoprocto longo e levemente bilobado; parâmeros curtos, digitiformes; edeago longo e delgado, afilando para o ápice.

Ovipositor (Fig. 9). Quando retraído, cerca de três vezes mais longo que o esternito 7 e com $2,8 \mathrm{~mm}$ de comprimento.
Pupa. Comprimento do corpo: 2,6 mm ( $\mathrm{n}=1)$. Cabeça (Fig. 11). Chifres antenais curtos; cerda apical longa com $0,12 \mathrm{~mm}$ de comprimento; três pares de papilas faciais laterais (um par com cerda curta e dois nus); dois pares de papilas faciais inferiores (um par com cerda longa, 0,08 mm de comprimento e um par nu). Tórax. Espiráculo protorácico digitiforme, longo, com $0,17 \mathrm{~mm}$ de comprimento. Cobertura das asas estendendo-se até a margem distal do terceiro segmento abdominal; cobertura das pernas anteriores, médias e posteriores alcançando, respectivamente o quinto distal do quinto segmento

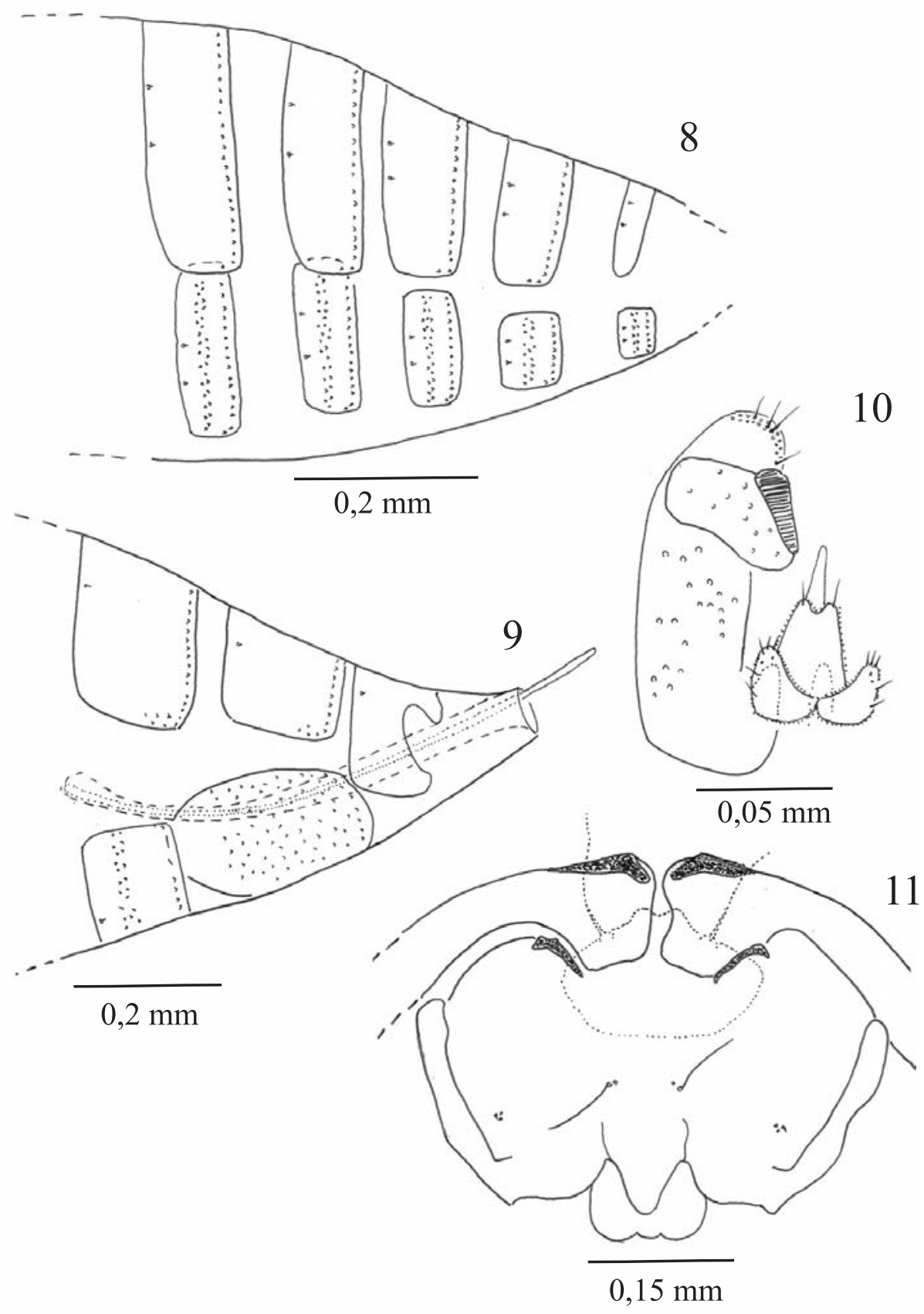

Figs. 8-11. Schizomyia spherica sp. nov.: 8, macho, segmentos abdominais 4 a 8, vista lateral; 9, fêmea, segmento abdominal 6 ao ápice do ovipositor, vista lateral; 10, terminália masculina, vista dorsal; 11, pupa, cabeça, vista frontal. 
abdominal, o terço basal do quinto segmento abdominal e o quinto basal do sexto segmento abdominal. Abdome (Fig. 12). Segmentos 3-8 com espinhos dorsais conspícuos, de tamanho variado distribuídos, na metade basal, apenas na parte discal, seguidos por inúmeros espinhos diminutos por todo o restante do segmento, exceto no quarto distal, onde há esculturas tegumentares. Alguns espinhos bífidos no ápice. Segmentos 3 a 6 com 9 a 11 espinhos grandes; segmentos 7, 8 e 9 com respectivamente 7, 7 e 3 espinhos grandes. Espiráculos abdominais curtos.
Larva. Coloração abóbora; corpo cilíndrico, levemente afilado na extremidade posterior. Comprimento: 1,4-1,9 mm ( $\mathrm{n}=4)$. Tegumento rugoso. Espátula protorácica com 0,17 mm de comprimento, dentes apicais afastados entre si e haste longa (Fig. 13), dois grupos de três papilas laterais de cada lado da espátula (em cada grupo: duas papilas providas de cerda com $0,01 \mathrm{~mm}$ de comprimento e uma papila nua), segmento terminal com quatros pares de papilas pouco desenvolvidas (três pares com cerda muito curta) (Fig. 14).
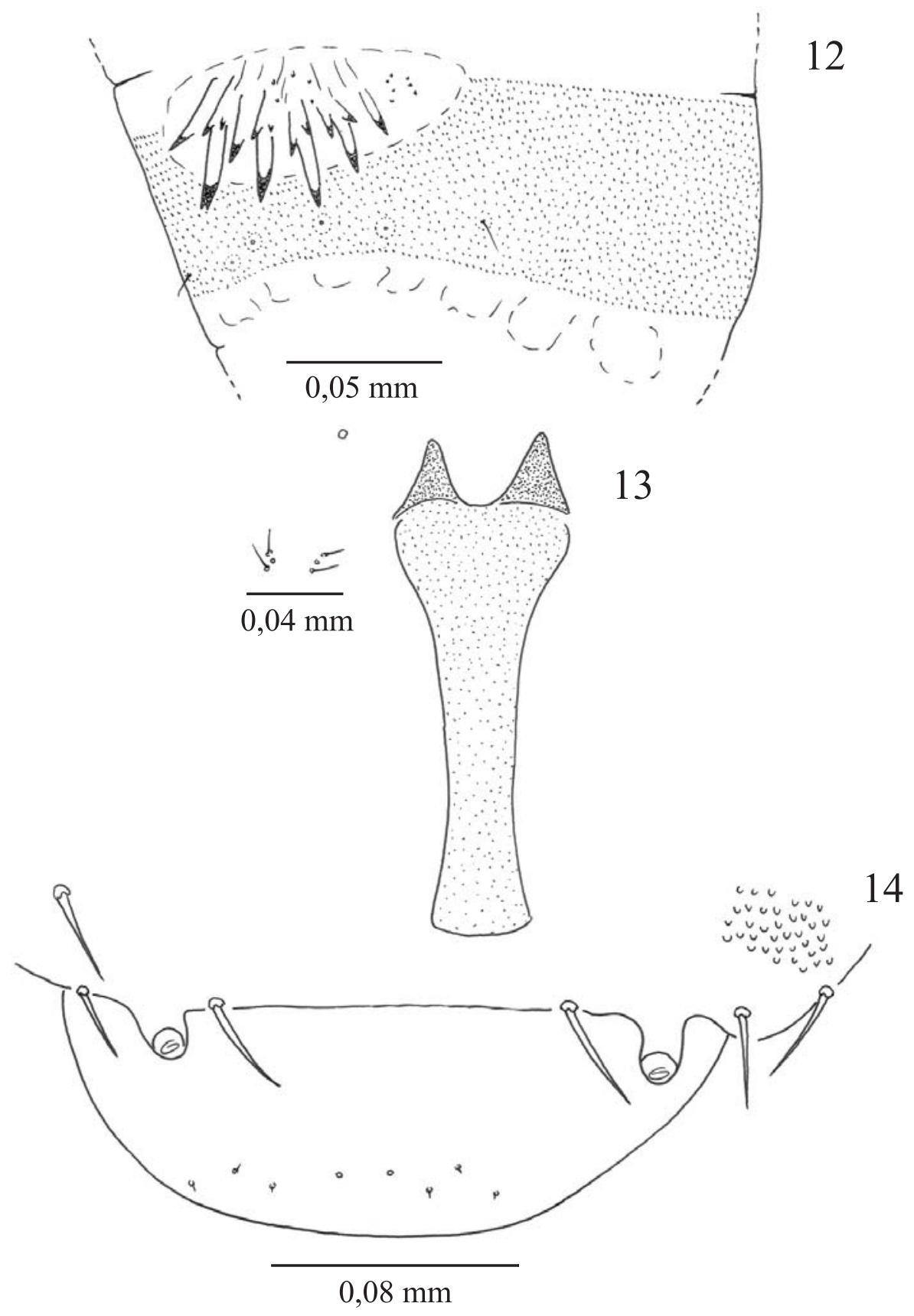

Fis. 12-14. Schizomyia spherica sp. nov.: 12, pupa, segmento abdominal 8, vista dorsal; 13, larva, espátula protorácica, papilas esternal e laterais, vista ventral; 14, larva, segmentos 8 e 9 , vista dorsal. 
Material examinado. Holótipo §’. BRASIL, Rio de Janeiro: Maricá (Restinga da Barra de Maricá), 20. IX.2004, Maia \& Azevedo leg. (MNRJ). Parátipos: mesma localidade do holótipo, $2 \circlearrowleft$ 08.IX.1998, V. Maia leg.; 8 larvas, 23.X.2000, V. Maia leg.; 4 \&, 1 exúvia pupal, 20.IX.2004, Maia \& Azevedo leg.; 8 \%, 16.VIII.2005.

U. Oliveira leg.; 4 larvas, 1 exúvia pupal, 06.IX.2005, Maia \& Oliveira leg.; 1 larva, 13.I.2006, Maia \& Oliveira leg. (MNRJ). Obtidos de galhas esféricas em Sebastiania glandulosa (Euphorbiaceae) (fig. 36, MAIA, 2001).

Além da espécie galhadora, foram identificados adultos de Lestodiplosis sp. (Diptera, Cecidomyidae), gênero com larvas predadoras principalmente de outras larvas de Cecidomyiidae e de ácaros. Material examinado: BRASIL, Rio de Janeiro: Maricá (Restinga da Barra de Maricá ), 8 \%, 3 \&, 20. IX.2004, Maia \&Azevedo leg. (MNRJ).

Etimologia. O epíteto específico refere-se à forma esférica da galha.

Comentários. Schizomyia spherica sp. nov. é a segunda espécie galhadora descrita para Sebastiania glandulosa. Ela difere de todas as demais espécies do gênero por apresentar os seguintes caracteres: papilas laterais com cerdas longas; espinhos dorsais na pupa com arranjo único; gonóstilo do macho com forma peculiar e ovipositor cerca de três vezes mais longo que o esternito 7.

\section{REFERÊNCIAS BIBLIOGRÁFICAS}

Barnes, H. F. 1932. Notes on Cecidomyiidae. Annals and Magazine of Natural History 10(9):475-484.

Felt, E. P. 1910. Schizomyia ipomoeae. Entomological News 21:160-161.

Gagné, R. J. 1994. The gall midges of the Neotropical region. Ithaca, Cornell University. 352p.

MAIA, V. C. 2001. The gall midges (Diptera, Cecidomyiidae) from three restinga of Rio de Janeiro State, Brazil. Revista Brasileira de Zoologia 18(2):583-629.

Maia, V. C. \& Fernandes, G. W. 2005. Two new species of Asphondyliini (Diptera: Cecidomyiidae) associated with Bauhinia brevipes (Fabaceae) in Brazil. Zootaxa 1091:27-40.

MöHN, E. 1960. Gallmucken (Diptera, Itonididae) aus El Salvador. 2. Teil. Senckenbergiana Biologica 41:197-240.

TAvares, J. S. 1925. Nova contribuição para o conhecimento da cecidologia brazileira. Brotéria, Série Zoológica, 22:97-98.

Urso-Guimarães, M. V. \& Amorim, D. S. 2002. New Brazilian species of Asphondyliini (Diptera: Cecidomyiidae). Revista Brasileira de Entomologia 46(4):561-570. 